\title{
MULTISCALE MODELING OF HEAT TREATMENT PROCESSING FOR SINGLE-CRYSTAL Ni- BASE SUPERALLOYS
}

\author{
Chen Shen ${ }^{1}$, Akane Suzuki ${ }^{1}$, Douglas G. Konitzer ${ }^{2}$ \\ ${ }^{1}$ GE Global Research, 1 Research Circle, Niskayuna, NY 12309 \\ ${ }^{2}$ GE Aviation, 1 Neumann Way, Cincinnati OH 45215
}

Keywords: Homogenization modeling, Microsegregation, $\gamma^{\prime}$ precipitation modeling, Single crystal, René N515

\begin{abstract}
A multi-component diffusion model coupled with CALPHAD thermodynamic and kinetics data was applied to simulate homogenization heat treatment for René N515 on a 2D crosssection. Different from conventional 1D models, spatial dendritic microstructures were explicitly taken into account. The predicted microsegregation evolution agreed well with EPMA experimental data. Incipient melting and topologically closed packed (TCP) phases were simulated. The effects of spatial microstructure and $\gamma^{\prime}$ dissolution were found to be a main reason for deviation of homogenization kinetics from a linear form of $\ln \delta \sim t$, derived from diffusion equation. $\gamma^{\prime}$ precipitation was simulated for René N515 under aging conditions. The predicted $\gamma^{\prime}$ coarsening was found in good agreement with experiment. The precipitation model can be combined with homogenization modeling to correlate heat treatment, residual microsegregation, and $\gamma^{\prime}$ microstructure.
\end{abstract}

\section{Introduction}

Heat treatment processing after casting of single-crystal (SX) superalloys is to both reduce solidification microsegregation and achieve a uniform distribution of $\gamma^{\prime}$ precipitates with optimal size and morphology. This process includes solution and aging steps. During solution, initial microsegregation is minimized, accompanied by dissolution of primary $\gamma^{\prime}$ and eutectic phases in interdendritic regions. The propensity of TCP phase formation in regions with segregated refractory elements is also reduced. During the subsequent cooling and aging steps, $\gamma^{\prime}$ precipitates are nucleated and continuously coarsened to a desirable size and spatial morphology.

Multiple physical processes are involved. Diffusion carries out redistribution of segregated chemical elements over a distance of hundreds of microns, of the typical size of the primary dendrite arm spacing, while $\gamma^{\prime}$ precipitation occurs at a smaller length scale of a few microns. Other types of phase transformation also occur, such as dissolution of interdendritic phases and re-melting (incipient melting) if the solution temperature or the initial heating rate is too high. Clearly, multiple length scales are involved in the heat treatment processing of SX alloys. Even during solution, element diffusion across primary dendrite arm spacing (PDAS) and secondary dendrite arm spacing (SDAS) can result in different time scales for the change in residual microsegregation. It is also noted that SX superalloys are highly alloyed with many elements, and that chemical interactions among the elements play significant roles in diffusion and phase transformations.

At present, an optimal heat treatment processing for SX alloys is mostly determined from experimental trials [1, 2], with systematically varied solution temperatures, steps, heating rates, cooling rates, and aging temperatures and duration. Each new processing variable adds considerably more effort on heat treatment, microstructure characterization, and material cost. For this application, analytical modeling has found great use. A simple solution to the diffusion equation, in a form of $\ln \delta \sim t$, has often been used to approximate the experimental homogenization data (segregation index $\delta$ ) in a post mortem analysis [3]. Due to the lack of an adequate basis of thermodynamic and kinetic data, it has very limited forward prediction capabilities, for example, to consider an alternative heat treatment temperature or a different alloy composition, without a significant amount of supporting experimental data. The recent CALPHAD based computational models have paved the road to fundamentally address alloy composition linkage. With commercial softwares such as DICTRA [4], people can predict formation of microsegregation and subsequent homogenization kinetics for a commercial alloy [5]. Such simulations are usually done in a simplified spatial configuration. For example, DICTRA only treats homogenization with a one-dimension (1D) model across the SDAS.

Variable length scales in microstructural evolution can manifest in experimental data. An analytical scaling behavior is often seen to be shifted (for example, in a slope of a diagram). During a homogenization process, for example, the governing diffusion distance for the change of microsegregation can shift continuously from SDAS to PDAS. Addressing real microstructural effects together with alloy composition is an important step toward a quantitative and predictive modeling capability for superalloy design and process development. In this paper, an integrated model for multi-step heat treatment processing of SX superalloys is presented, with an explicit account of solidification microstructure, alloy composition, and realistic non-isothermal heat treatment conditions. Predictions of residual microsegregation, $\gamma^{\prime}$ precipitation, and process related phenomena such as incipient melting can provide guidance to design of a process before it actually begins. A multiscale simulation of homogenization and $\gamma^{\prime}$ precipitation for a second generation SX alloy will be presented as a case study.

\section{Modeling homogenization of microsegregation}

\section{Homogenization model}

Long-range diffusion is the principal kinetic process that occurs during homogenization. The driving force is the compositional gradient between dendrite core and interdendritic region developed during solidification. The gradient is over a length scale on the order of primary dendritic arm spacing. In a simple form, by solving Fick's second law of diffusion for a single-phase binary alloy at a fixed temperature, one can write the residual microsegregation as a function of time [6]

$$
\delta=\exp \left(-\frac{4 \pi^{2} D}{\lambda^{2}} t\right)
$$


with $t$ the heat treatment time, $D$ the diffusion coefficient, and $\lambda$ the diffusion distance. The dimensionless quantity, $\delta=$ $\left[c^{\max }(t)-c^{\min }(t)\right] /\left[c^{\max }(0)-c^{\min }(0)\right]$, is defined as the ratio of the composition difference at $t$, to its initial state. When (1) is strictly applicable, experimental data plotted in a diagram of $\ln \delta$ versus $t$ would appear in a straight line.

Homogenization of a superalloy involves simultaneous diffusion of all the chemical elements. Instead of using the composition gradient, the driving force is modeled more generally by the chemical potential gradient of each element. The corresponding multi-component diffusion equation becomes [7,8] $\partial c_{i} / \partial t=$ $-\nabla \cdot J_{i}$, with $i$ indexing each element, and the diffusion flux $J_{i}=-M_{i j} \nabla \mu_{j}$ (a summation over the index $j$ is implied). The chemical potential $\mu_{j}$ and chemical mobility $M_{i j}$ can be modeled in the CALPHAD framework with suitable thermodynamic and mobility databases. Since more than one solid phase may present in a superalloy (e.g., primary $\gamma^{\prime}$ ), depending on solution temperature and the starting microsegregation, we adopt a multiphase treatment by Larsson et al $[9,10]$. The above single-phase diffusion flux is replaced by an effective flux $\widetilde{J}_{l}=-\widetilde{M}_{i j} \nabla \tilde{\mu}_{j}$ that combines contributions from individual phases [9]. Interactions among diffusion species and phases are automatically accounted for. In this work, the model has been implemented in a general 3D computing code for studying effects of microstructure spatial variation. The code can run $1 \mathrm{D}, 2 \mathrm{D}$ or $3 \mathrm{D}$ simulations. When the initial composition input is given as a $2 \mathrm{D}$ or $3 \mathrm{D}$ map, from either experiment or simulation, spatial effects of actual dendrite microstructure are incorporated. The code is linked to ThermoCalc through the TQ-I interface for low-level thermodynamic and mobility calculations.

In this study, the model was applied to a second-generation SX alloy René N515 (Ni-7.5Co-6Cr-2Mo-6.25W-1.5Re-6.3Al-6.5Ta$0.6 \mathrm{Hf}-0.035 \mathrm{C}-0.004 \mathrm{~B}$, wt. $\%$; Ni-7.7Co-7Cr-1.3Mo-2.05W0.49Re-14Al-2.2Ta-0.2Hf-0.18C-0.02B, at. \%). All alloy elements, except for the interstitial species $\mathrm{B}$ and $\mathrm{C}$, were treated in the simulation. For simplicity, only the two primary solid phases, $\gamma$ and $\gamma^{\prime}$, were included. Quantitative EPMA maps of all the nine elements, measured in an area of $1.2 \times 1.2 \mathrm{~mm}^{2}$ of a transverse section of directionally solidified single-crystal René N515 specimen, were provided as the initial condition ("Ascast"). The model simulated the subsequent homogenization process on a 2D plane (Figure 1). The heat treatment included an initial heat-up rate and then an isothermal solution at $1293{ }^{\circ} \mathrm{C}$ for up to 60 hours. The simulation snapshots are shown in Figure 1.

Al

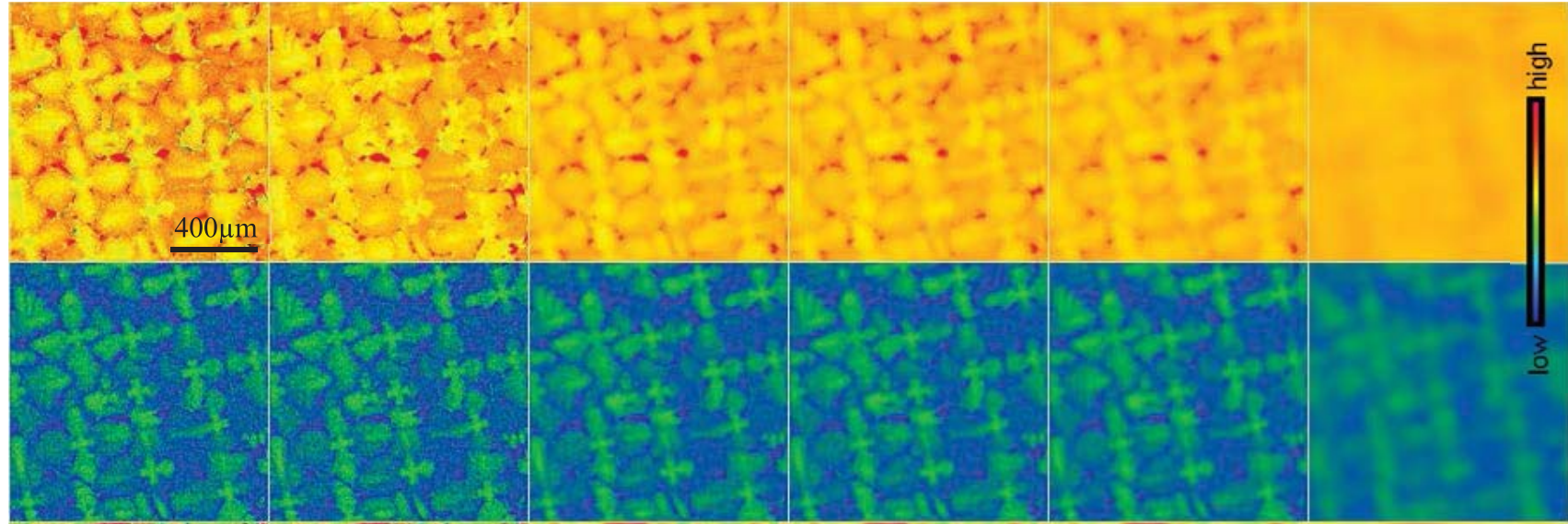

$\mathbf{W}$

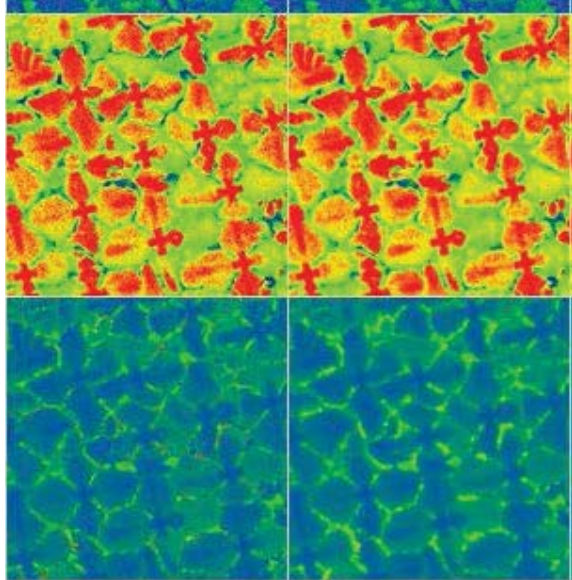

As-cast

Reach $1271{ }^{\circ} \mathrm{C}$

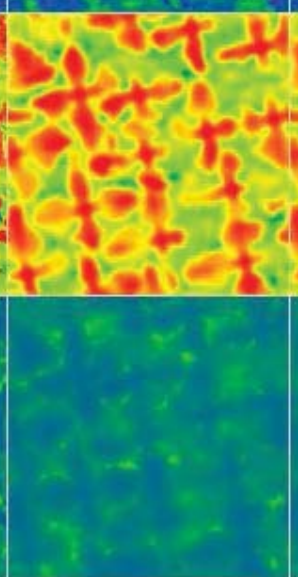

Reach $1293{ }^{\circ} \mathrm{C}$
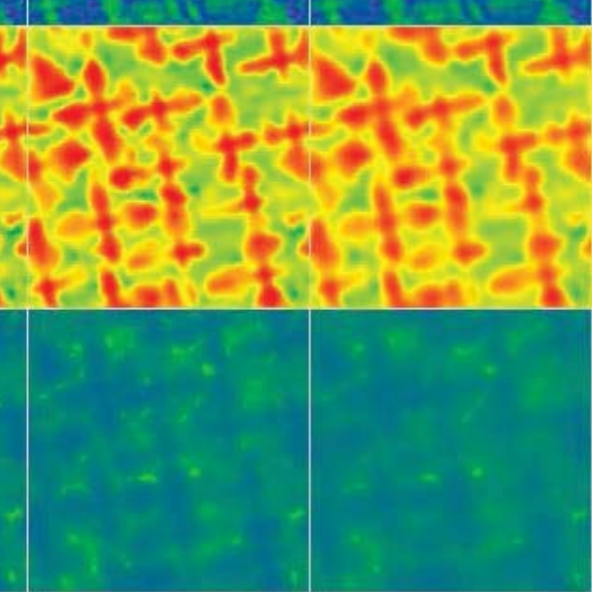

$1293{ }^{\circ} \mathrm{C} / 1 \mathrm{hr}$

$1293{ }^{\circ} \mathrm{C} / 4 \mathrm{hrs}$

$1293{ }^{\circ} \mathrm{C} / 60 \mathrm{hrs}$

Figure 1: Simulation of homogenization of René N515. Four selected elements are shown. "As-cast" is experimentally obtained map, the other maps are from the simulation. The snapshots during heating up to $1293^{\circ} \mathrm{C}$ and isothermal solution are shown. 


\section{Quantify microsegregation}

Two kinds of difficulties were encountered when we tried to quantify microsegregation from a real microstructure. (1) Spatial variation of the dendrite structure led to variation of interdendritic composition. Local interdendritic areas such as 'A' in Figure 2(a) were often seen with a large deviation of compositions from a typical interdendritic composition. (2) Interdendritic particles such as MC carbides interfered with reliable quantification of interdendritic composition. These stable phases have large element partitioning such as high Ta, and very low Al. Although these particles generally do not participate in homogenization, the strong degree of elemental partitioning would add an apparent composition variation if not excluded properly.

To resolve these difficulties three quantification methods were compared:

Method-a: with (1) in mind, use full EPMA map (Figure 2(a)), sort the data in an ascending or descending order. Take the extreme (maximal and minimal) values as dendrite core and interdendritic compositions

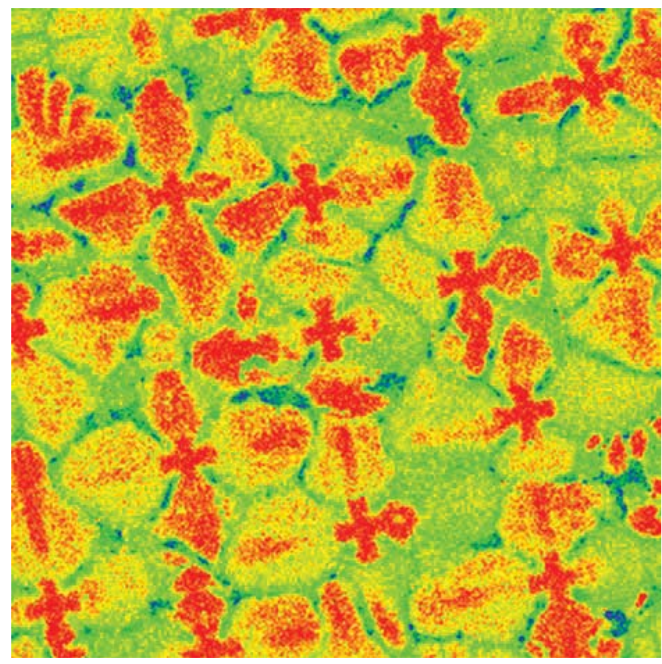

(a)

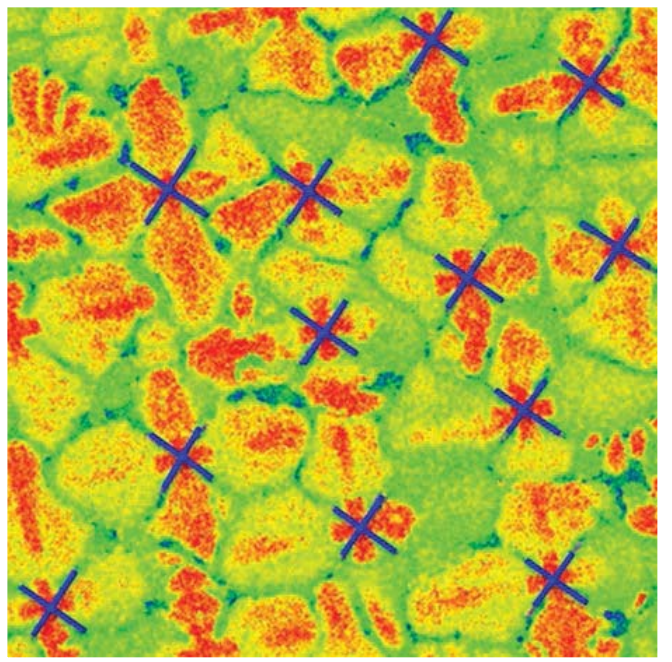

(c)
Method-b: consider (2), down-sampling the full map data on a coarse grid (Figure 2(b)) in order to reduce the chance of encountering interdendritic particles while still able to capture spatial variation of composition. Take the extreme values as dendrite core and interdendritic compositions

Method-c: manually select line profiles across dendrites (Figure 2(c)), to completely avoid interdendritic particles. Multiple lines give a typical (averaged) dendritic profile

The comparison in Figure 2(d) and Figure 3 suggests that the full EPMA map generally over counts dendrite core and interdendritic compositions, and that the coarse grid data vs. manual profile gives consistent dendrite core compositions while the interdendritic compositions from Method-b are more prone to the effects of spatial variations. Overall, coarse grid data provides a good measure of actual microsegregation with much less influence from interdendritic particles, while manual profiles give a more averaged result.

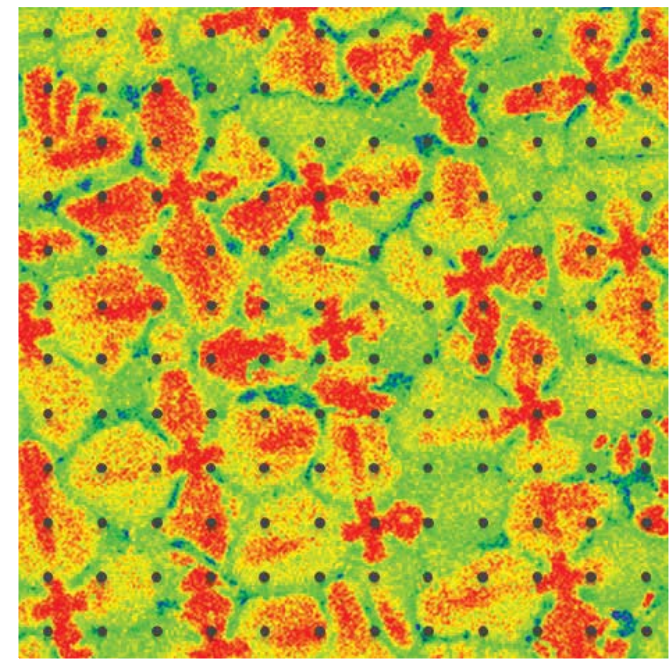

(b)

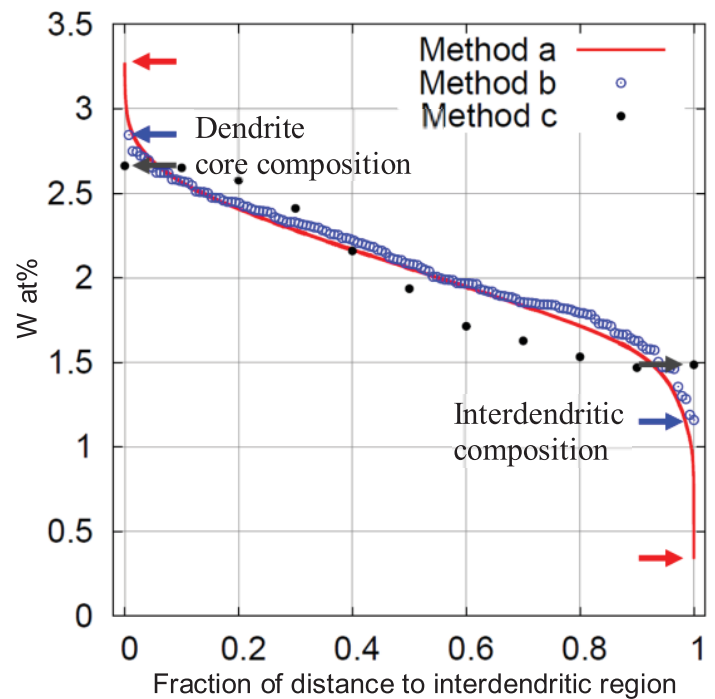

(d)

Figure 2: Three methods for quantifying microsegregation (shown for W): (a) use full EPMA map, (b) down-sample on a coarse grid, (c) manually select profiles across dendrite cores. The results are compared in (d). The dendrite core and interdendritic compositions identified by each method are marked by the arrows in (d). 


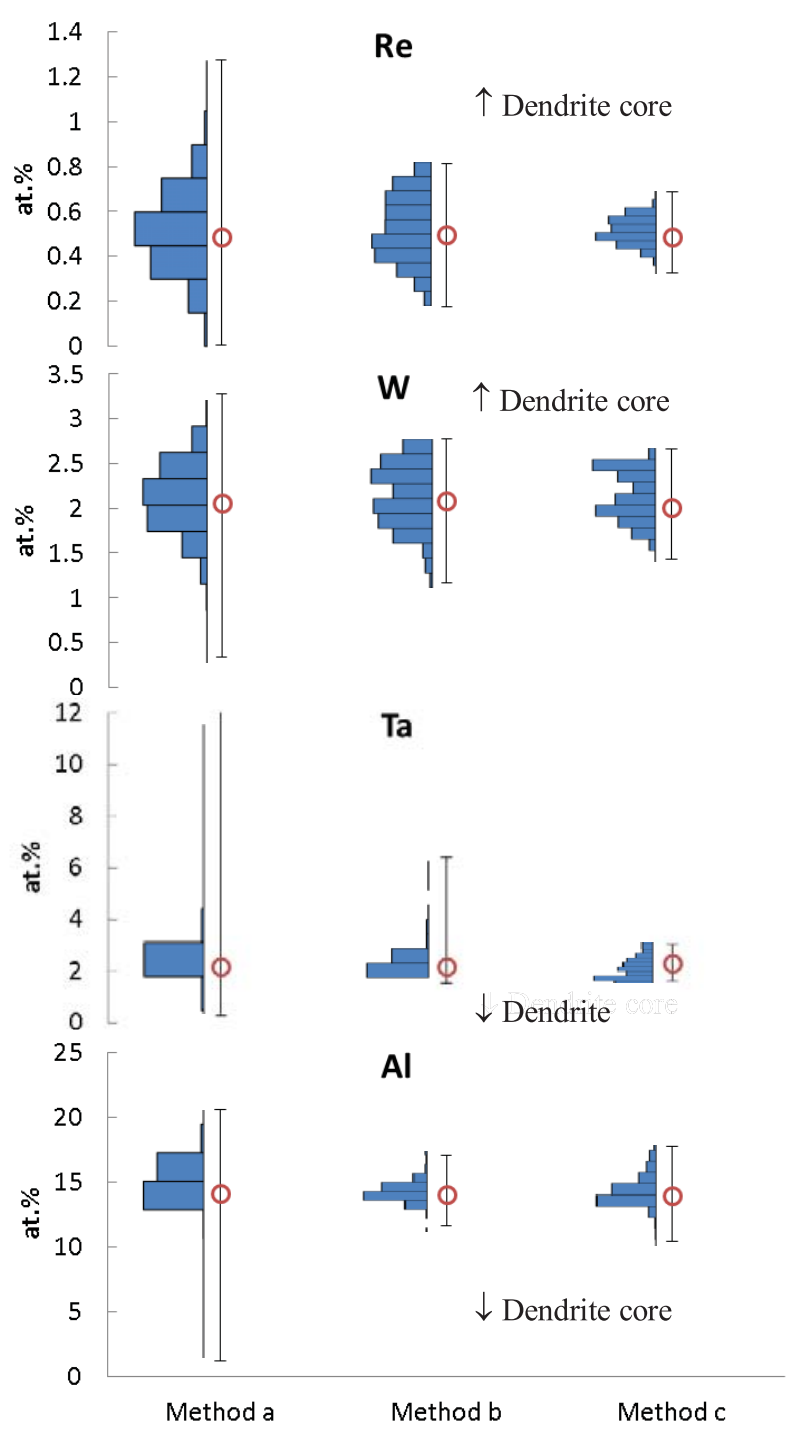

Figure 3: Comparison of the three methods for quantifying microsegregation in as-cast René N515 (Vertical bars: maximum and minimum compositions, circles: average composition. A histogram is given on the left side.).

\section{$\underline{\text { Comparison of simulation with experiment }}$}

The methods described above were applied to analyze the simulation results in Figure 1. It is found (see Figure 4):

(1) When applied to the simulation data, differences between the full map method (dotted lines) and the coarse grid method (dashed lines) diminished quickly. Because no third-phase particles were considered in the simulation, the large localized composition heterogeneities due to interdendritic MC particles in the input EPMA data were quickly relaxed through diffusion (although in the real material they still remain). The difference between the coarse grid method (blue dashed line) and the manual profile method (blue solid lines) are mostly shown to the interdendritic composition. For $\mathrm{Al}$, the difference was found also contributed by undissolved primary $\gamma^{\prime}$.

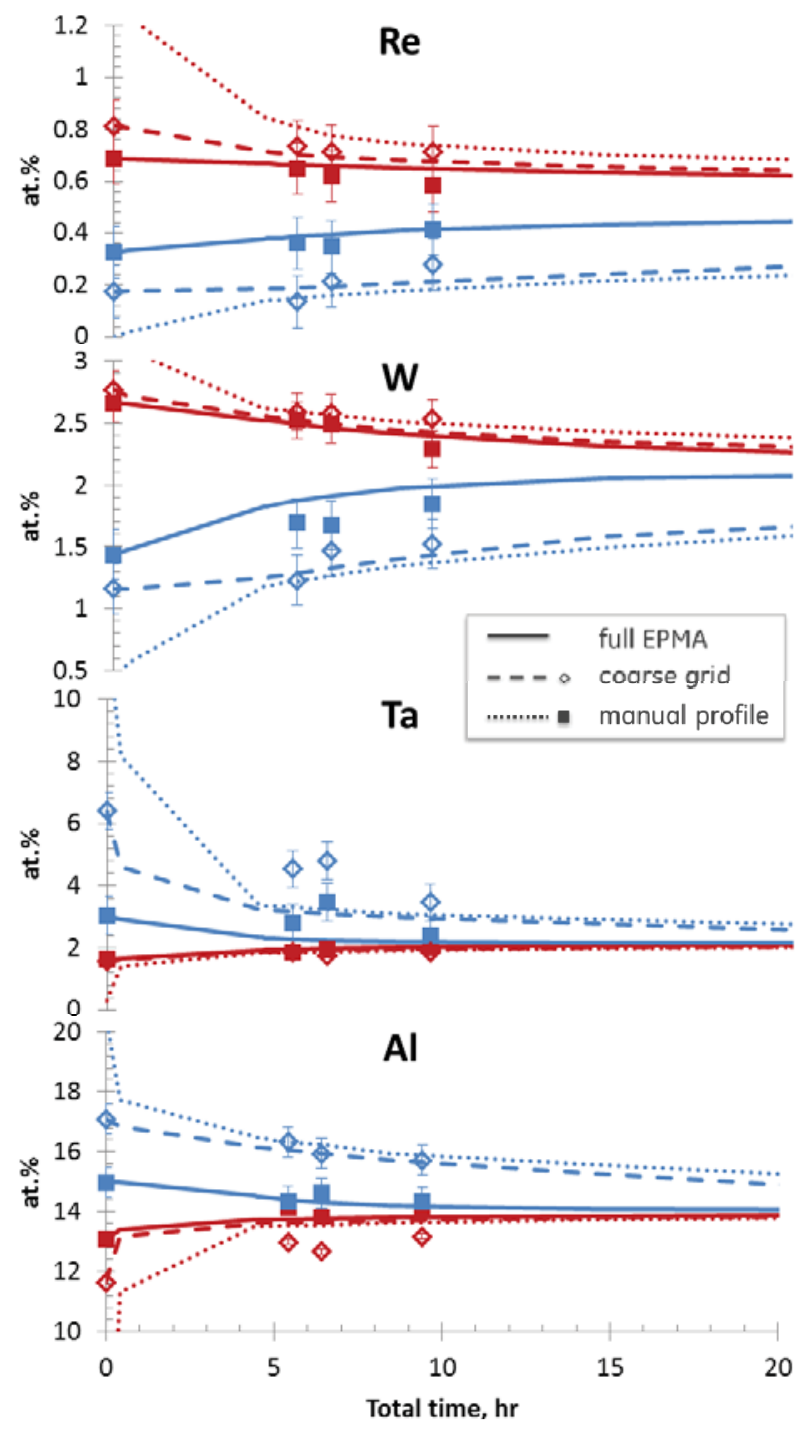

Figure 4: Comparison of simulation (lines) and experiment (symbols) for René N515 homogenization. Red and blue colors represent dendrite core and interdendritic compositions, respectively. The total time includes an initial heat up time of about 5 hours.

(2) By applying either Method-b (dashed lines vs. open diamonds) or Method-c (solid lines vs solid squares), the simulation is found to agree well with EPMA experimental data for most elements during homogenization. The large discrepancy of Ta and $\mathrm{Hf}$ interdendritic compositions was found due to interdendritic particles captured in the EPMA data.

\section{Prediction of TCP phases}

Segregation of refractory elements in dendrite cores increases the risk of local TCP phase formation that causes degradation to the alloy's mechanical properties. Since the refractory elements typically exhibit limited diffusion, their homogenization rate is a limiting factor for a solution heat treatment. To evaluate TCP phase formation in a given heat treatment process, we took intermediate outputs of composition maps from the homogenization simulation in Figure 1, and performed 
equilibrium phase calculations through ThermoCalc TQ-I with TTNI7 database [11]. At $1093{ }^{\circ} \mathrm{C}$, for example, the main TCP phase in René N515 alloy was predicted to be $\mu$ phase at a volume fraction of $0.04 \%$. Figure 5 shows that after a short 1 hour solution at $1293{ }^{\circ} \mathrm{C}$, the $\mu$ phase can form as high as $1.8 \%$ volume fraction in dendrite core. The amount of $\mu$ phase decreases with homogenization time. Even after a long solution time of 60 hours it still remains at about $0.8 \%$, due to residual microsegregation of $\mathrm{Re}$ and $\mathrm{W}$ (see also Figure 4). It should be noted that the predictions in Figure 5 only gave an upper-bound estimate because the actual kinetic times for TCP phase formation were ignored. A more rigorous treatment can be made by combining TCP precipitation kinetic modeling such as in the recent development [12, 13].

\section{Predict incipient melting and optimize heat treatment}

A solution heat treatment often starts with a slow heating up step to avoid local melting in interdendritic regions (incipient melting). During heating, the diffusion process starts to be activated. Homogenization of the initial microsegregation, especially in the interdendritic regions, results in a small increase in the incipient temperature. With a sufficiently slow heating rate the increase of incipient temperature is allowed to catch up with the increase in heat treatment temperature, without causing the material to melt. Therefore, predicting the evolution of incipient temperature is closely relevant to a heat treatment design.

Using the intermediate composition outputs from the homogenization simulation, a spatial map of melting temperature can be calculated in a similar way as for the TCP phase calculation: at each 'pixel' in the map, local composition is used to calculate the temperature at which liquid phase starts to form. The lowest melting temperature in the map (' $M$ ' in Figure 6(a)) is then regarded as the incipient melting temperature at that time. Repeating the same procedure for different times produces a plot of the incipient temperature with respect to heat treatment time (Figure 6(b)). As homogenization proceeds, the incipient melting temperature increases, and the solution temperature can be raised. In general this process is a function of the heat treatment condition. Iterating the simulation along with selected heat treatment variables can be used to design an optimal heat treatment process.

\section{Effect of microstructure on homogenization rate}

If the time evolution of microsegregation at a fixed temperature simply follows equation (1), a plot of $\ln \delta$ will be linear to $t$; or equivalently, a plot of $\ln [-\ln \delta]$ vs $\ln t$ will be a straight line with a slope of 1 . Analyzing a series of $2 \mathrm{D}$ isothermal simulation results indicated that none of the nine elements actually followed such a trend. Figure 7(a) shows, for element $\mathrm{W}$, that $\ln \delta$ vs. $t$ generally exhibits a concaved shape instead of a straight line, which means a slower homogenization kinetics than predicted by equation (1) at a later time. Accordingly, the slope of $\ln [-\ln \delta]$ vs $\ln t$ is shown close to 0.7 for $\mathrm{W}$, instead of 1 (Figure 7(b)). Similar behaviors are seen for all the other elements, although the slopes differ.

Examining the difference between the current model and the analytical model (equation (1)) leads to the following possible causes:
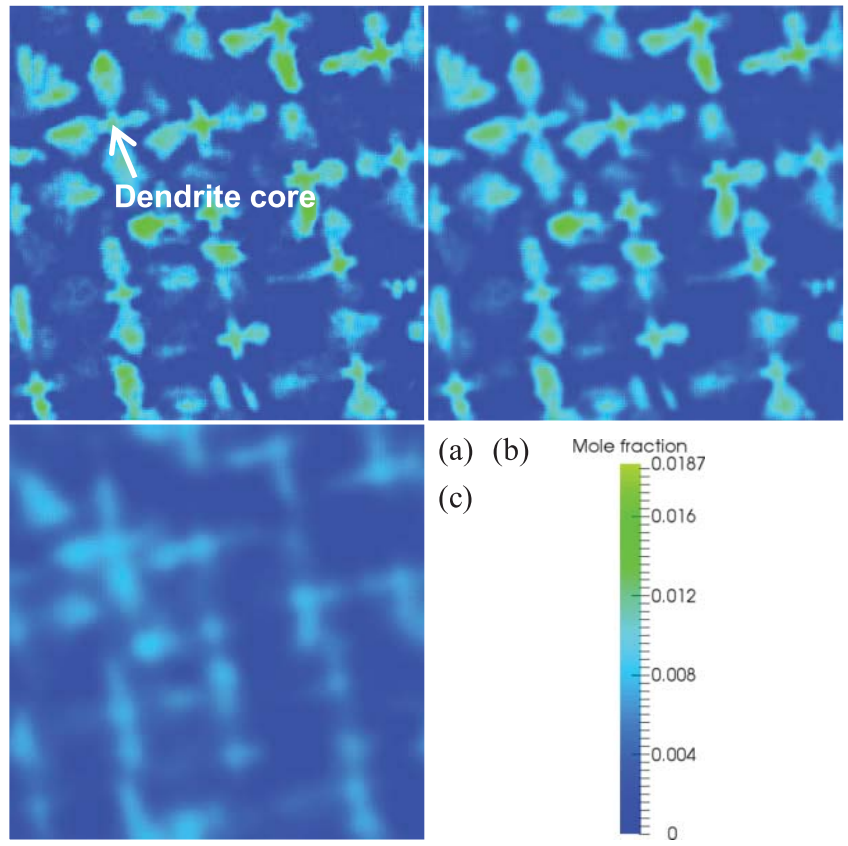

Figure 5: Predicted mole fraction of $\mu$ phase in René N515 at $1093{ }^{\circ} \mathrm{C}$ after solution at $1293{ }^{\circ} \mathrm{C}$ for (a) $1 \mathrm{hr}$, (b) $4 \mathrm{hrs}$, and (c) 60 hrs.

(a)
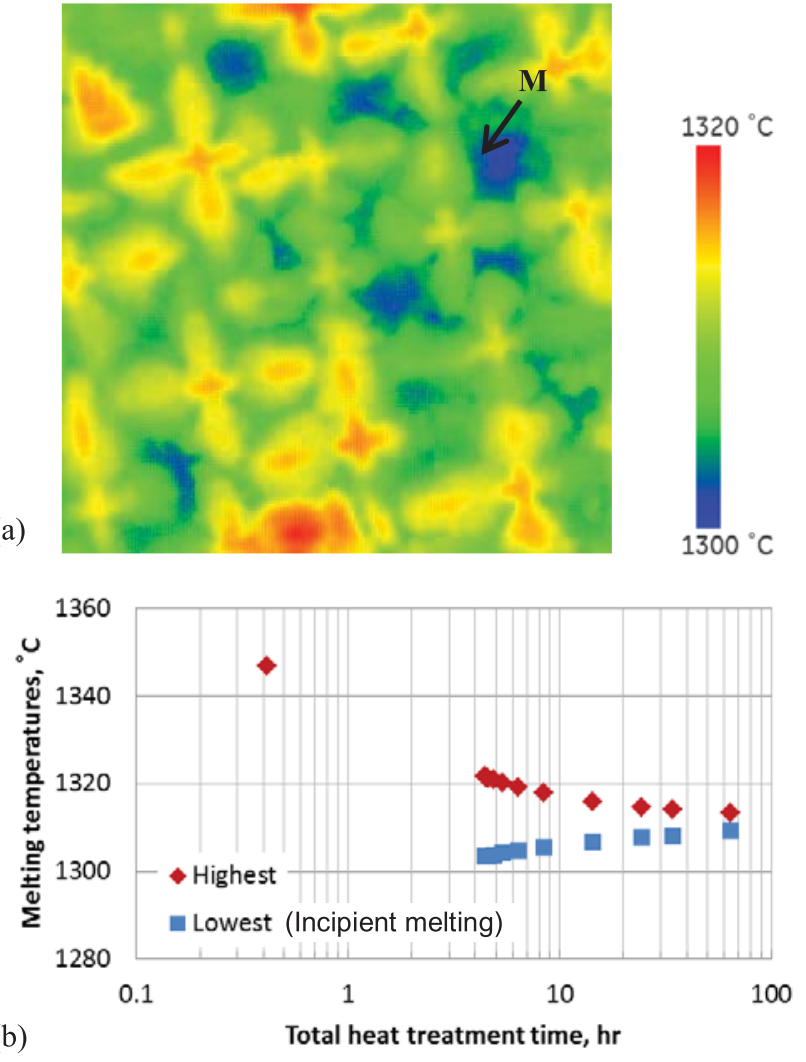

Figure 6: Predicted incipient melting: (a) melting temperature distribution after $1293{ }^{\circ} \mathrm{C} / 1 \mathrm{hr}$, (b) incipient melting temperature increases with time. 
- Chemistry effect (elemental interactions in multicomponent diffusion)

- Spatial effect (microstructure affecting diffusion distance)

- $\quad \gamma^{\prime}$ effect (undissolved $\gamma^{\prime}$ affecting chemical potential of elements)

To isolate the main factor, four additional sets of simulation were performed, each done at two temperatures 1093 and $1316{ }^{\circ} \mathrm{C}$ :

(1) $2 \mathrm{D}$ simulation of binary composition $\mathrm{Ni}-\mathrm{X}$, with $\mathrm{X}=\mathrm{Al}$ or $\mathrm{W}$. The initial composition of $\mathrm{X}$ is taken the same as in Figure 1, while other elements are substituted by Ni. The same dendritic microstructure is retained. Note the compositions resulted in single $\gamma$ phase at both 1093 and $1316^{\circ} \mathrm{C}$ from the very beginning

(2) $1 \mathrm{D}$ simulation of full multicomponent composition, with the averaged EPMA profile by Method-c used as initial composition. $\gamma / \gamma^{\prime}$ two phase at $1093{ }^{\circ} \mathrm{C}$, while single $\gamma$ phase at $1316{ }^{\circ} \mathrm{C}$ with initial $\gamma^{\prime}$ dissolution

(3) $1 \mathrm{D}$ simulation similar to (2), except that the initial composition is given in a sine wave form with the maximum and minimum matched to the EPMA profile. $\gamma / \gamma^{\prime}$ two phase at $1093{ }^{\circ} \mathrm{C}$, while single $\gamma$ phase at $1316{ }^{\circ} \mathrm{C}$ with initial $\gamma^{\prime}$ dissolution

(4) $1 \mathrm{D}$ simulation of binary composition $\mathrm{Ni}-\mathrm{X}$, with $\mathrm{X}=\mathrm{Al}$ or $\mathrm{W}$. The compositions resulted in single $\gamma$ phase at both 1093 and $1316{ }^{\circ} \mathrm{C}$ from the very beginning

Comparisons of these results in Figure 8 and Figure 9 lead to the following observations:

- Reducing chemistry to binary composition, but with the same 2D spatial dendrite microstructure, did not fundamentally change the deviation of slope of $\ln [-\ln \delta]$ vs. $\ln t$. Thus the chemistry effect was not the main effect.

- Reducing spatial configuration to 1D but with the full multi-component composition showed a noticeable change of the slope toward the theoretical value 1 .

- Additionally, using a sine wave form as the initial composition profile brought the slope even closer to 1 . These suggest a significant effect of 2D spatial microstructure, and to a lesser degree the shape of the $1 \mathrm{D}$ profile, on the homogenization behavior.

- The $\gamma^{\prime}$ phase had strong effects on the $\gamma^{\prime}$ forming element $\mathrm{Al}$. At $1316{ }^{\circ} \mathrm{C}$ the slopes changed significantly when $\gamma^{\prime}$ was dissolving. At $1093{ }^{\circ} \mathrm{C}$, where $\gamma^{\prime}$ is stable, long term trend of $\mathrm{Al}$ deviated from slope 1. The $\gamma^{\prime}$ effect to $\mathrm{W}$ was much less in all cases.

A simulation on a longitudinal 2D section along the solidification direction was also performed in comparison with the simulation on the transverse section such as in Figure 1. The main difference was found that on the longitudinal cross-section, the homogenization rate was faster in the primary solidification direction, in which the much narrower SDAS dictated the diffusion distance. In comparison, on the transverse section, homogenization was all dominated by PDAS and took longer time to achieve a similar residual microsegregation level.

Overall, it was seen that the spatial dendritic microstructure has significant effects on homogenization kinetics. The effects act mainly via diffusion distance, such as $\lambda$ in equation (1), which governs diffusion gradient at a given level of microsegregation.
Rather than staying constant, the diffusion distance continuously increased during homogenization and gradually shifted from SDAS to PDAS. Such a change led to a time-dependent behavior of the homogenization rate. From a practical point of view, the slow end of homogenization rate may be an important factor for evaluating the effectiveness of a heat treatment design.
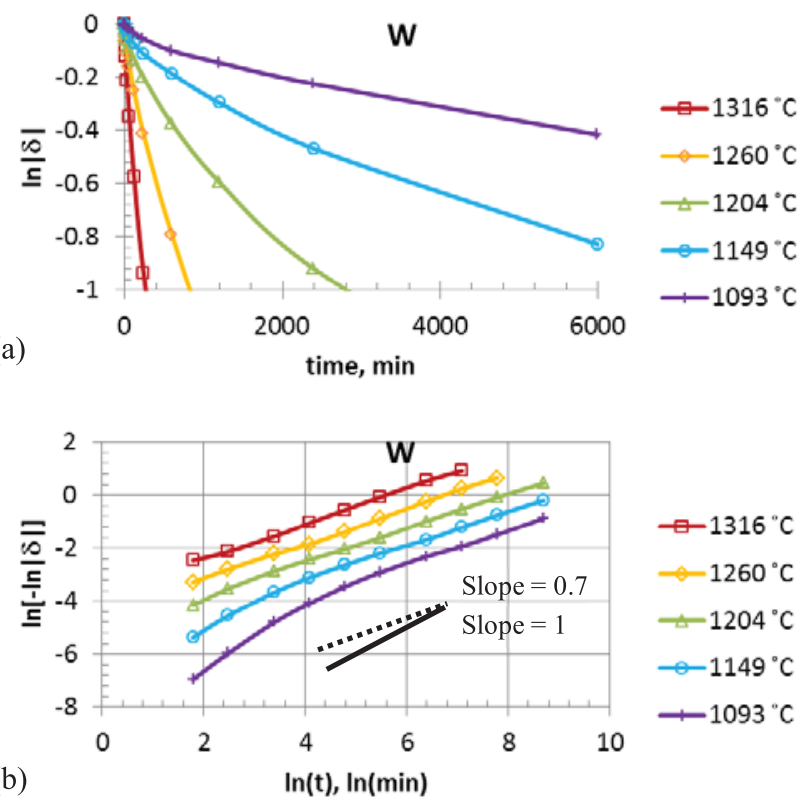

Figure 7: Results from 2D isothermal simulations suggested that homogenization kinetics did not follow equation (1) (W is shown as an example).

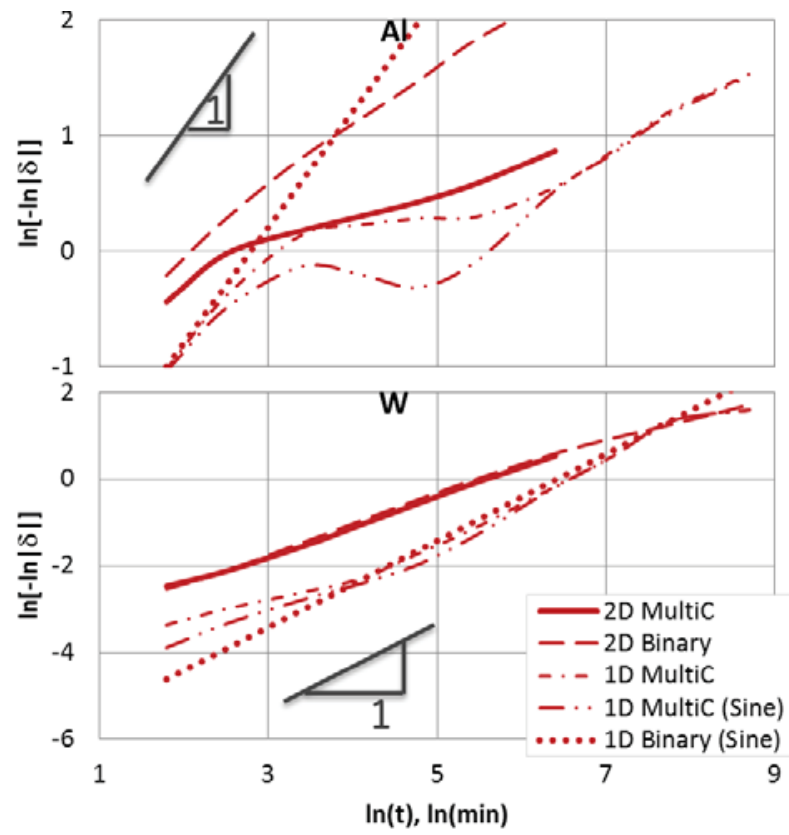

Figure 8: Comparison of homogenization of $\mathrm{Al}$ and $\mathrm{W}$ from five simulation configurations at $1316{ }^{\circ} \mathrm{C}$. The $2 \mathrm{D}$ multicomponent simulation "2D MultiC" is same as in Figure 7. The "1D Binary" clearly reproduces the theoretic slope of 1 . 


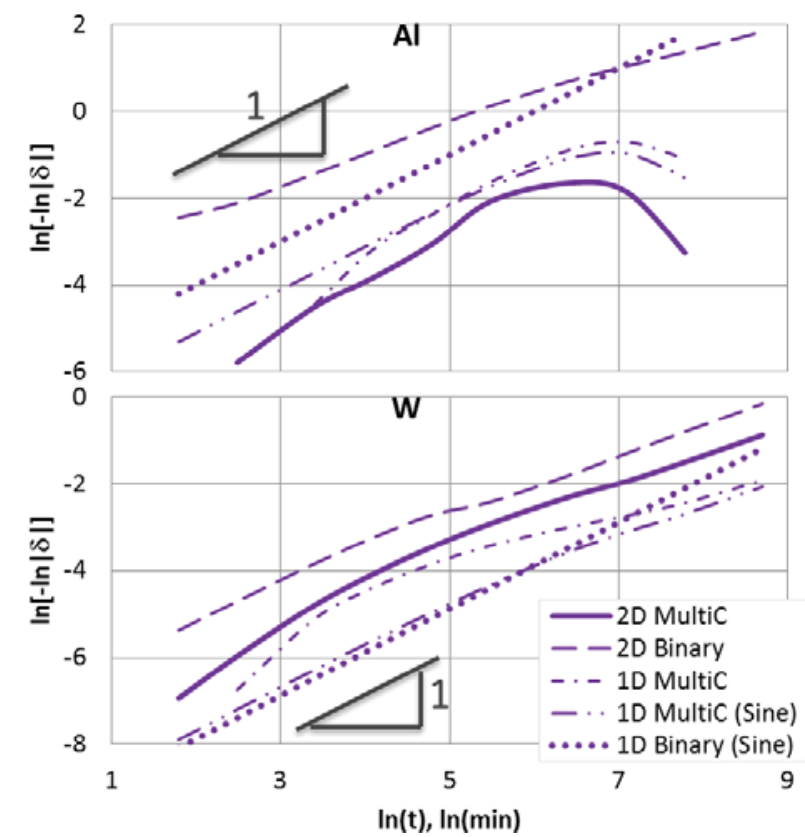

Figure 9: Comparison of homogenization of $\mathrm{Al}$ and $\mathrm{W}$ from five simulation configurations at $1093{ }^{\circ} \mathrm{C}$. The $2 \mathrm{D}$ multicomponent simulation "2D MultiC" is same as in Figure 7. The "1D Binary" clearly reproduces the theoretic slope of 1 .

\section{Modeling $\gamma^{\prime}$ microstructure}

After solution, aging heat treatments are applied to develop $\gamma^{\prime}$ microstructure in SX alloys. Optimal $\gamma^{\prime}$ size, shape, and spatial distribution are critical to an alloy's high temperature mechanical properties, such as creep $[14,15]$. The combination of cooling rate, aging temperature and time determines the $\gamma^{\prime}$ microstructure. In particular, the cooling from solution temperature controls nucleation and the initial size and number density of $\gamma^{\prime}$; and aging steps control coarsening and development of desirable cuboidal shape and spatial alignment of the precipitates.

A Langer-Schwartz type precipitation model [16-18] was applied to model the response of $\gamma^{\prime}$ size and volume fraction to a general non-isothermal heat treatment cycle for René N515. The full alloy composition was used in ThermoCalc calculations, with TTNI7 and MOBNI1 databases, to generate thermodynamic driving forces for nucleation and growth, and diffusivity data. For computational efficiency, the data were reduced to a pseudobinary description [19], in which $\gamma^{\prime}$ forming elements ( $\mathrm{Al}, \mathrm{Ti}, \mathrm{Ta}$, $\mathrm{Nb}$ ) were lumped to form an equivalent element for $\gamma^{\prime}$ and the rest to form an equivalent element for the matrix $\gamma$ phase. The reduction from multi-component composition to pseudo-binary description has been automated, so that $\gamma^{\prime}$ modeling could be linked to homogenization modeling for assessing effects of residual microsegregation to $\gamma^{\prime}$ formation. Two calibration parameters, $\gamma / \gamma^{\prime}$ interfacial energy and a diffusion correction constant were used for René N515. The activation energy of diffusion was found to be about $284 \mathrm{~kJ} / \mathrm{mol}$.

A set of aging heat treatment experiments were performed (Figure 10 (a)) at 1093,1149 , and $1204{ }^{\circ} \mathrm{C}$. At each temperature condition, specimens were taken out of the furnace at different times. An ice brine quench was used to retain the $\gamma^{\prime}$ microstructures for each

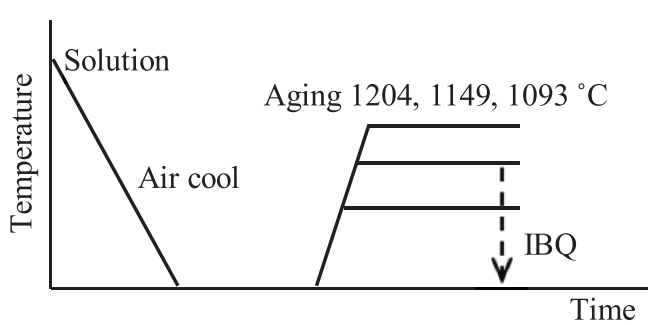

(a)

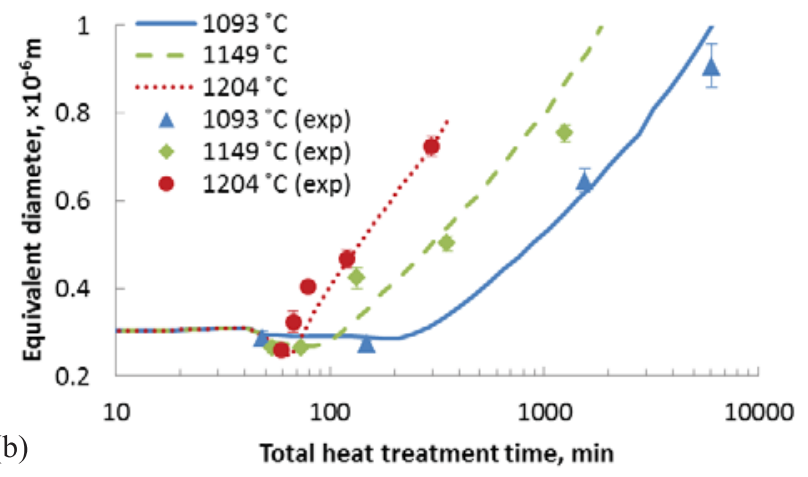

Figure 10: Simulation of $\gamma^{\prime}$ sizes for René N515: (a) schematic of the heat treatment cycles, (b) comparison of simulation (lines) and experimental data (symbols).

aging temperature. For each specimen, scanning electron microscopy (SEM) images were taken in the dendrite core region at two randomly selected locations. $\gamma^{\prime}$ sizes were measured from the SEM images at two magnifications between 5,000X and $25,000 X$. In some specimens with long aging times, large coalesced $\gamma^{\prime}$ particles were excluded from size analysis. Figure 10(b) compares the average $\gamma^{\prime}$ sizes (converted to equivalent spherical diameters) between simulation and experiment. The error bars on the experimental data are the standard deviation of the averaged sizes from four images $(2$ areas and 2 magnifications) per specimen. The model predicted reasonably well the $\gamma^{\prime}$ aging kinetics for all the three temperature conditions. Since the model was developed for general non-isothermal conditions, it can be used to simulate more complex heat treatment paths, such as multi-step aging.

\section{Summary}

A multi-component diffusion model coupled with CALPHAD thermodynamic and kinetics data was applied to simulate the homogenization heat treatment for René N515 on a 2D crosssection. Without any parameter fitting, the predicted evolution of microsegregation agreed with experimental EPMA data, especially for Re and W. Incipient melting, TCP phases, and the effects of microstructural spatial variation were studied.

It was observed that the homogenization rate slowed down with time due to a gradual shift of the characteristic diffusion distance from SDAS to PDAS. This highlights one of the important effects of spatial dendritic microstructure on homogenization process, which is not normally accounted for in 1D models. Dissolution of $\gamma^{\prime}$ was found to strongly influence the homogenization of $\mathrm{Al}$, Ti, and also to $\mathrm{Cr}$ and Mo in René N515, but much less to Re, W, and $\mathrm{Ta}$. These factors were found to cause deviation of the trend of homogenization rate from a commonly used form $\ln \delta \sim t$. 
The $\gamma^{\prime}$ precipitation was simulated for René N515. The alloy composition was incorporated with the use of CALPHAD databases, and for efficiency was reduced to a pseudo-binary description for various thermodynamic driving forces and diffusivities in the model Compared with aging experiments, the model predicted reasonably well the $\gamma^{\prime}$ coarsening kinetics at 1093,1149 , and $1204{ }^{\circ} \mathrm{C}$ aging for René N515. The precipitation model can be combined with homogenization modeling to correlate heat treatment, residual microsegregation, and $\gamma^{\prime}$ microstructure.

\section{Acknowledgement}

M.P. Knussman, D.A. Wark, R.L. Casey, D.Y. Wei, M.E. Krug, W-J Zhang, S.J. Duclos, M.L. Blohm, PR Subramanian, S.V. Thamboo are gratefully acknowledged for technical and program support.

\section{References}

1. G.E. Fuchs, "Solution heat treatment response of a third generation single crystal Ni-base superalloy". Materials Science and Engineering: A. 300(1-2)(2001), 52-60.

2. S.R. Hegde, R.M. Kearsey, and J.C. Beddoes, "Designing homogenization-solution heat treatments for single crystal superalloys". Materials Science and Engineering: A. 527(2122)(2010), 5528-5538.

3. Z.-j. Miao, et al., "Quantitative analysis of homogenization treatment of INCONEL718 superalloy". Transactions of Nonferrous Metals Society of China. 21(5)(2011), 1009-1017.

4. A. Borgenstam, et al., "DICTRA, a tool for simulation of diffusional transformations in alloys". Journal of Phase Equilibria. 21(3)(2000), 269-280.

5. P.D. Jablonski and C.J. Cowen, "Homogenizing a NickelBased Superalloy: Thermodynamic and Kinetic Simulation and Experimental Results". Metallurgical and Materials Transactions B. 40(2)(2009), 182-186.

6. T.Z. Kattamis and M.C. Flemings, "Segregation in castings". Transactions of AIME. 233(1965), 992-999.

7. J. Ågren, "Diffusion in phases with several components and sublattices". Journal of Physics and Chemistry of Solids. 43(5)(1982), 421-430.

8. J.O. Andersson and J. Ågren, "Models for numerical treatment of multicomponent diffusion in simple phases". Journal of Applied Physics. 72(4)(1992), 1350-1355.

9. H. Larsson and A. Engström, "A homogenization approach to diffusion simulations applied to $\alpha+\gamma \mathrm{Fe}-\mathrm{Cr}-\mathrm{Ni}$ diffusion couples". Acta Materialia. 54(9)(2006), 2431-2439.

10. H. Larsson and L. Höglund, "Multiphase diffusion simulations in 1D using the DICTRA homogenization model". Calphad. 33(3)(2009), 495-501.

11. TTNI7. Available from: http://www.thermotech.co.uk/ databases.html\#Ni-DATA.
12. R. Rettig and R.F. Singer, "Numerical modelling of precipitation of topologically close-packed phases in nickel-base superalloys". Acta Materialia. 59(1)(2011), 317-327.

13. R. Rettig and R.F. Singer. "Influence of ruthenium on topologically close packed phase precipitation in single-crystal nibased superalloys: numerical experiments and validation". in Superalloys 2012: 12th International Symposium on Superalloys. 2012. Seven Springs, PA, USA: TMS (The Minerals, Metals \& Materials Society).

14. S. Steuer, et al., "Creep behavior under isothermal and nonisothermal conditions of AM3 single crystal superalloy for different solutioning cooling rates". Materials Science and Engineering: A. 601(2014), 145-152.

15. R.A. MacKay, T.P. Gabb, and M.V. Nathal, "Effects of Microstructural Parameters on Creep of Nickel-Base Superalloy Single Crystals", in NASA Report NASA/TM-2013-217868. 2013.

16. R. Kampmann and R. Wagner, in Decomposition of alloys: the early stages, P. Haasen, et al., Editors. 1984, Pergamon Press: Oxford. p. 91-103.

17. J.S. Langer, "Theory of nucleation rates". Physical Review Letters. 21(14)(1968), 973-976.

18. h. Wendt and P. Haasen, "Nucleation and growth of $\gamma^{\prime}$ Precipitates in Ni-14 at.\% Al". Acta Metallurgica. 31(10)(1983), 1649-1659.

19. T.P. Gabb, et al. " $\gamma$ ' formation in a nickel-base disk superalloy". in Superalloys 2000. 2000: TMS. 